\title{
ADAPTIVE EMERGENCY BRAKING CONTROL WITH OBSERVER-BASED DYNAMIC TIRE/ROAD FRICTION MODEL AND UNDERESTIMATION OF FRICTION COEFFICIENT ${ }^{1}$
}

\author{
Luis Alvarez ${ }^{*, 2}$ Jingang $\mathbf{Y i}^{* *}$ Roberto Horowitz ${ }^{* *}$ \\ Luis Olmos* \\ * Instituto de Ingeniería, Universidad Nacional Autónoma de \\ México, 04510 Coyoacán DF, México \\ ** Department of Mechanical Engineering, University of \\ California, Berkeley, CA 94720-1740, USA
}

\begin{abstract}
An adaptive control scheme for emergency braking of vehicles is designed based on a LuGre dynamic model for the tire/road friction. The wheel angular speed and longitudinal vehicle acceleration information are used to design an observerestimator for vehicle velocity, friction internal state and friction parameters. A Lyapunov-based stabilizing controller is designed to achieve near maximum braking capability of the vehicle. Underestimation of the friction coefficient is guaranteed by a proper choice of adaptation gains and initial conditions of the friction parameters. Simulation results show that both the state estimation and the friction parameters converge to the true values and that vehicle stops rapidly.
\end{abstract}

Keywords: Automotive control, adaptive control, nonlinear observers.

\section{INTRODUCTION}

Important research has been undertaken in recent years to investigate safety in both manual traffic and Automated Highway Systems (AHS) when, in an attempt to increase traffic flow, highway densities are significantly increased. One specific issue that greatly impacts overall safety is the influence of the tire/road interaction on the braking capabilities of vehicles during emergency braking manuevers.

Research in tire/road friction modeling and estimation for individual vehicles is abundant. The pseudo-static model given in (Bakker et al., 1987), known as the "magic formula", gives a good approximation to experimental results and is widely used in automotive research and industries. How-

\footnotetext{
1 Research supported by UCB-ITS PATH grant MOU-373 and UNAM-DGAPA PAPIIT grant IN-104700.

2 Corresponding author, alvar@pumas.iingen.unam.mx.
}

ever, this model has a complex analytical structure and its parameters are difficult to identify. In (Kiencke, 1993) and (Alvarez et al., 2000) identifiable pseudo-static parametric friction models are presented. Although the parameters in these models lack direct physical interpretation, they can be identified through on-line adaptation.

Dynamic friction models (Canudas et al., 1995) were introduced to capture the friction phenomenon more accurately. In (Canudas de Wit and Tsiotras, 1999) a LuGre model was modified to replicate the tire/road interface. This model was used in (Canudas de Wit and Horowitz, 1999) to estimate the tire/road friction coefficient under different road conditions, and was applied in an adaptive braking controller in (Yi et al., 2001).

The goal of this paper is to extend the results of (Yi et al., 2001). Assuming that the parameters of the Lugre model are unknown and the wheel 
angular velocity and vehicle longitudinal acceleration measurable, a parameter adaptation law is proposed that overcomes the slow convergence problem in (Yi et al., 2001). Underestimation of the friction coefficient, a very desirable feature from the safety point of view, is guaranteed by the proper choice of the parameter adaptation gains and initial conditions of the estimated parameters.

\section{SYSTEM DYNAMICS}

This paper considers only the longitudinal dynamics of the vehicle. It is assumed that the four wheels of the vehicle apply the same braking force,that the road has no slope and that the weight of the vehicle is distributed evenly among the four wheels. A quarter vehicle model is used and a modified lumped LuGre friction model is considered as follows:

$$
\begin{gathered}
\dot{z}=v_{r}-\frac{\sigma_{0}\left|v_{r}\right|}{h\left(v_{r}\right)} z \\
J \dot{\omega}=r F_{x}-u_{\tau} \\
m \dot{v}=-4 F_{x}-F_{a}
\end{gathered}
$$

where $z$ is the friction internal state, $v_{r}=v-$ $r \omega$ is the relative velocity, $h\left(v_{r}\right)=\mu_{c}+\left(\mu_{s}-\right.$ $\left.\mu_{c}\right) e^{-\left|\frac{v_{r}}{v_{s}}\right|^{1 / 2}}, \mu_{s}$ is the normalized static friction coefficient, $\mu_{c}$ is the normalized Coulomb friction, $v_{s}$ is the Stribeck relative velocity, $u_{\tau}$ is the traction/braking torque, $F_{x}$ the traction/braking force given by the tire/road contact, $F_{a}$ the aerodynamic force, $m$ the vehicle mass, $J$ the tire rotational inertia, and the parameter $\sigma_{0}$ models the rubber longitudinal stiffness. The braking force $F_{x}$ is given by

$$
F_{x}=F_{n}\left(\sigma_{0} z+\sigma_{1} \dot{z}+\sigma_{2} v_{r}\right)
$$

where $\sigma_{1}$ is the rubber longitudinal damping, $\sigma_{2}$ is the viscous relative damping and $F_{n}=m g / 4$. By (Wong, 1993), the aerodynamic force can be modeled as

$$
F_{a}=C_{a v} v^{2} .
$$

Substitute the Eq. above into (1c) and consider $v_{r}=v-r \omega$ as the state variable and then rewrite Eqs. (1b) and (1c) as

$$
\begin{aligned}
\dot{v} & =-c \mu-d v^{2}, \\
\dot{v}_{r} & =-(a+c) \mu-d v^{2}+e K_{b} P_{b},
\end{aligned}
$$

with $a=r^{2} m g / 4 J, c=g, d=C_{a v} / m$ and $e=$ $r / J$. As suggested in (Gerdes and Hedrick, 1995), the braking torque is approximated by $\tau_{b}=K_{b} P_{b}$, where $K_{b}$ is an overall braking system gain and $P_{b}$ the master cylinder pressure.

\section{COMPENSATOR DESIGN}

\subsection{Velocity observer}

Assuming that the wheel angular velocity and acceleration and the longitudinal acceleration are known, the instantaneous value of $\mu$ is derived from Eq. (1b) and it is possible to propose the following observer for the velocity

$$
\dot{\hat{v}}=-c \mu-d \hat{v}^{2}+L \tilde{y}_{2}
$$

where $\tilde{y}_{2}:=\dot{v}-\hat{\dot{v}}=-d \tilde{v}(v+\hat{v})$ with $\hat{\dot{v}}:=-c \mu-$ $d \hat{v}^{2}$.

The velocity estimation error dynamics are

$$
\dot{\tilde{v}}=-d \tilde{v}(v+\hat{v})(1-L)
$$

Define the Lyapunov candidate function

$$
W_{1}=\frac{1}{2} \tilde{v}^{2}
$$

Its time derivative is

$$
\dot{W}_{1}=\tilde{v} \dot{\tilde{v}}=-d \tilde{v}^{2}(v+\hat{v})(1-L) \leq 0
$$

Introduce the following lemma

Lemma 1. Assume $L<0$, then $\tilde{v}(0)<0 \Rightarrow \tilde{v}(t)<$ $0, \forall t \geq 0$ or $\tilde{v}(0)>0 \Rightarrow \tilde{v}(t)>0, \forall t \geq 0$.

Proof: For any given value of $v$ and $\hat{v}$ the solution to Eq. (5) is of the form

$$
\tilde{v}(t)=\tilde{v}(0) e^{-(1-L) \int_{0}^{t} d(v+\hat{v}) d \tau}
$$

This term will never change sign, therefore if $\tilde{v}(0)<0 \Rightarrow \tilde{v}(t)<0, \forall t \geq 0$ or if $\tilde{v}(0)>0 \Rightarrow$ $\tilde{v}(t)>0, \forall t \geq 0$

Remark 2. Lemma 1 implies $\dot{W}_{1}<0$ and asymptotic stability of $\tilde{v}=0$ follows. Moreover, if the observer gain $|L|$ is chosen large, the estimated velocity $\hat{v}$ converges to the true value $v$ quickly.

\subsection{Internal state observer and adaptive parameter estimations}

Substituting Eq. (1a) into Eq. (2)

$$
\begin{aligned}
\mu & =\sigma_{0} z+\sigma_{1}\left[v_{r}-\sigma_{0} f\left(v_{r}\right) z\right]-\sigma_{2} v_{r} \\
& =\sigma_{0} z-\sigma_{3} f\left(v_{r}\right) z+\sigma_{4} v_{r}
\end{aligned}
$$

where $\sigma_{3}=\sigma_{0} \sigma_{1}$ and $\sigma_{4}=\sigma_{1}-\sigma_{2}$. This expression is linear in the parameters $\sigma_{0}, \sigma_{3}$ and $\sigma_{4}$, i.e.,

$$
\mu=\mathbf{U} \Theta
$$

where $\mathbf{U}:=\left[\begin{array}{lll}z & -f\left(v_{r}\right) z & v_{r}\end{array}\right]$ and $\Theta:=$ $\left[\begin{array}{lll}\sigma_{0} & \sigma_{3} & \sigma_{4}\end{array}\right]^{T}$.

For the internal state $z$, propose the observer

$$
\dot{\hat{z}}=\hat{v}_{r}-\hat{\sigma}_{0} f\left(\hat{v}_{r}\right) \hat{z}
$$

and a gradient type parameter adaptation law

$$
\dot{\tilde{\Theta}}=-\Gamma \hat{\mathbf{U}}^{T} \tilde{\mu}
$$

where $\Gamma=\operatorname{diag}\left(\gamma_{0}, \gamma_{3}, \gamma_{4}\right)>0$ is a diagonal matrix of adaptation gains, $\hat{\mathbf{U}}$ is the regressor in 
Eq. (9) evaluated at the estimated quantities, i.e. $\hat{\mathbf{U}}=\left[\begin{array}{lll}\hat{z}-f\left(\hat{v}_{r}\right) \hat{z} & \hat{v}_{r}\end{array}\right]$ and $\tilde{\mu}$ is defined by

$$
\tilde{\mu}=\mathbf{U} \Theta-\hat{\mathbf{U}} \hat{\Theta}=\hat{\mathbf{U}} \tilde{\Theta}+\tilde{\mathbf{U}} \Theta
$$

with $\tilde{\mathbf{U}}=\mathbf{U}-\hat{\mathbf{U}} \cdot \tilde{\mu}=\mu-\hat{\mu}$ is defined as the error of the friction coefficient ${ }^{3}$.

Developing Eq. (12)

$$
\begin{aligned}
\tilde{\mu}= & {\left[\sigma_{0}-\sigma_{3} f\left(v_{r}\right)\right] \tilde{z}+\hat{z} \tilde{\sigma}_{0}-f\left(\hat{v}_{r}\right) \hat{z} \tilde{\sigma}_{3}+\hat{v}_{r} \tilde{\sigma}_{4} } \\
& +\sigma_{4} \tilde{v}_{r}-\sigma_{3} \hat{z}\left[f\left(v_{r}\right)-f\left(\hat{v}_{r}\right)\right]
\end{aligned}
$$

If the term $f\left(v_{r}\right)-f\left(\hat{v}_{r}\right)$ is expanded in a Taylor series about $v_{r}$ and $\tilde{v}_{r}=v_{r}-\hat{v}_{r}=\tilde{v}$ is used, Eq. (13) can be rewritten as

$$
\begin{aligned}
\tilde{\mu}= & {\left[\sigma_{0}-\sigma_{3} f\left(v_{r}\right)\right] \tilde{z}+\hat{z} \tilde{\sigma}_{0}-f\left(\hat{v}_{r}\right) \hat{z} \tilde{\sigma}_{3}+\hat{v}_{r} \tilde{\sigma}_{4} } \\
& +\left[\sigma_{4}-\sigma_{3} \hat{z} f^{\prime}\left(v_{r}\right)\right] \tilde{v}
\end{aligned}
$$

with $f^{\prime}\left(v_{r}\right)=d f\left(v_{r}\right) / d v_{r}$.

The error dynamics of $\tilde{z}$ from Eqs. (1a) and (10) are given by

$$
\dot{\tilde{z}}=\left[1-\sigma_{0} f^{\prime}\left(v_{r}\right) \hat{z}\right] \tilde{v}-\sigma_{0} f\left(v_{r}\right) \tilde{z}-f\left(\hat{v}_{r}\right) \hat{z} \tilde{\sigma}_{0}
$$

\subsection{Controller design}

In this paper, a LuGre dynamic tire/road friction model will be used to estimate the maximum slip $\lambda_{m}$ by means of an equivalent pseudo-static model. Following (Yi et al., 2000), the following pseudo-static relationship between $\mu$ and $\lambda$ is obtained after solving a distributed LuGre tire/road friction model

$$
\begin{aligned}
& \mu=h\left(v_{r}\right)\left[1+2 \gamma \frac{h\left(v_{r}\right)}{\sigma_{0} l|\eta|}\left(e^{-\frac{\sigma_{0} l|\eta|}{2 h\left(v_{r}\right)}}-1\right)\right]+\sigma_{2} v_{r} \\
& \eta=\frac{v_{r}}{r \omega}=-\frac{\lambda}{1-\lambda}, \quad \gamma=1-\frac{\sigma_{1}|\eta|}{r \omega h\left(v_{r}\right)}
\end{aligned}
$$

where $l$ is the length of the tire/road contact patch.

To continue with the controller design, it is necessary to set the value for the pressure of the master cylinder, $P_{b}$; for that purpose define

$$
\tilde{s}=\hat{v}_{r}-\hat{\lambda}_{m} \hat{v}=\hat{v}\left(1-\hat{\lambda}_{m}\right)-r \omega
$$

as the desired relative velocity for the emergency braking maneuver. In this expression $\tilde{s}=\hat{v}_{r}-$ $r \omega$ and $\hat{\lambda}_{m}$ is the estimated value of $\lambda_{m}$ by Eq. (16) based on the current estimation of $\hat{\Theta}$ and $\hat{v}$. Taking the time derivative of Eq. (17)

$$
\begin{aligned}
\dot{\tilde{s}}= & \dot{\hat{v}}\left(1-\hat{\lambda}_{m}\right)-r \dot{\omega}-\hat{v} \dot{\hat{\lambda}}_{m}=\dot{\hat{v}}\left(1-\hat{\lambda}_{m}\right)-\frac{r f}{J} \mu \\
& +\frac{r e K_{b} P_{b}}{J}-\hat{v} \frac{\partial \hat{\lambda}_{m}}{\partial \hat{v}} \dot{\hat{v}}-\hat{v} \frac{\partial \hat{\lambda}_{m}}{\partial \omega} \dot{\omega} .
\end{aligned}
$$

\footnotetext{
3 Recall that the instantaneous friction coefficient $\mu$ is derived from Eq.(1b) under the assumption that the angular acceleration and the braking pressure can be measured.
}

The partial derivatives of $\lambda_{m}$ can be calculated numerically. Choosing

$$
\begin{aligned}
P_{b}= & \frac{J}{r e K_{b}}\left[-\dot{\hat{v}}\left(1-\hat{\lambda}_{m}\right)+\frac{r f}{J} \mu+\hat{v} \frac{\partial \hat{\lambda}_{m}}{\partial \hat{v}} \dot{\hat{v}}\right. \\
& \left.+\hat{v} \frac{\partial \hat{\lambda}_{m}}{\partial \omega} \dot{\omega}-\zeta \bar{s}\right]
\end{aligned}
$$

where $\zeta>0$ a gain and substituting in Eq. (18) gives

$$
\dot{\tilde{s}}=-\zeta \tilde{s} \text {. }
$$

Define the following Lyapunov function candidate

$$
W_{4}=\frac{1}{2} \tilde{s}^{2}
$$

Taking the time derivative of Eq. (21) and using Eq. (20)

$$
\dot{W}_{4}=-\zeta \tilde{s}^{2} \leq 0
$$

The asymptotic stability of $\tilde{s}=0$ follows.

\subsection{Combined stability analysis}

Propose, in addition to Eq. (6), the following set of Lyapunov function candidates

$$
W_{2}=\frac{1}{2} \tilde{z}^{2} ; W_{3}=\frac{1}{2} \tilde{\Theta}^{T} \Gamma^{-1} \tilde{\Theta}
$$

and define now the composite Lyapunov function candidate

$$
W=W_{1}+W_{2}+W_{3}=\sum_{i=1}^{3} W_{i}
$$

The time derivative of Eq. (24) can be written as

$$
\dot{W}=\tilde{v} \dot{\tilde{v}}+\tilde{z} \dot{\tilde{z}}+\tilde{\Theta}^{T} \Gamma^{-1} \dot{\tilde{\Theta}}
$$

Using the observer error dynamics and parameter adaptation law in Eqs. (5), (15) and (11), Eq. (25) becomes

$$
\begin{aligned}
\dot{W}= & -d(v+\hat{v})(1-L) \tilde{v}^{2}+\tilde{z}\left[\left(1-\sigma_{0} f^{\prime}\left(v_{r}\right) \hat{z}\right) \tilde{v}\right. \\
& \left.-\sigma_{0} f\left(v_{r}\right) \tilde{z}-f\left(\hat{v}_{r}\right) \hat{z} \tilde{\sigma}_{0}\right] \\
& -\left(\tilde{\Theta}^{T} \hat{\mathbf{U}}^{T} \hat{\mathbf{U}} \tilde{\Theta}+\tilde{\Theta}^{T} \hat{\mathbf{U}}^{T} \tilde{\mathbf{U}} \Theta\right)
\end{aligned}
$$

The term $\tilde{\mathbf{U}}$ can be expressed as

$$
\tilde{\mathbf{U}}=\mathbf{U}_{1} \tilde{v}+\mathbf{U}_{2} \tilde{z} \text {. }
$$

where $\mathbf{U}_{1}=\left[\begin{array}{lll}0 & -f^{\prime}\left(v_{r}\right) \hat{z} & 1\end{array}\right]$ and $\mathbf{U}_{2}=$

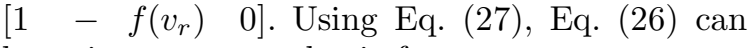
be written as a quadratic form

$$
\dot{W}=-\Phi^{T} \mathbf{M} \Phi
$$

where $\Phi=\left[\begin{array}{lll}\tilde{\Theta} & \tilde{z} & \tilde{v}\end{array}\right]^{T}=\left[\begin{array}{lllll}\tilde{\sigma}_{0} & \tilde{\sigma}_{4} & \tilde{\sigma}_{4} & \tilde{z} & \tilde{v}\end{array}\right]^{T}$,

$$
\mathbf{M}=\left[\begin{array}{ccccc}
\hat{z}^{2} & -\hat{z}^{2} \hat{f} & \hat{z} \hat{v}_{r} & w_{1} \hat{z} & w_{2} \hat{z} \\
-\hat{z}^{2} \hat{f} & \hat{z}^{2} \hat{f}^{2} & -\hat{z} \hat{f} \hat{v}_{r} & -w_{1} \hat{z} \hat{f} & -w_{2} \hat{z} \hat{f} \\
\hat{z} \hat{v}_{r} & -\hat{z} \hat{f} \hat{v}_{r} & \hat{v}_{r}^{2} & w_{1} \hat{v}_{r} & w_{2} \hat{v}_{r} \\
\hat{z} \hat{f} & 0 & 0 & \sigma_{0} f\left(v_{r}\right) & -w_{3} \\
0 & 0 & 0 & 0 & w_{4}
\end{array}\right]
$$

with $\hat{f}=f\left(\hat{v}_{r}\right), w_{1}=\sigma_{0}-\sigma_{3} f\left(v_{r}\right), w_{2}=\sigma_{4}-$ $\sigma_{3} f^{\prime}\left(v_{r}\right) \hat{z}, w_{3}=1-\sigma_{0} f^{\prime}\left(v_{r}\right) \hat{z}$ and $w_{4}=d(1-$ $L)(v+\hat{v})$. 
It is direct to show that $\mathbf{M} \geq 0$ by the fact that

$$
\operatorname{det} \mathbf{M}_{s}(1,1)=\hat{z}^{2}>0, \quad \operatorname{det} \mathbf{M}_{s}(1: j, 1: j)=0,
$$

where $\mathbf{M}_{s}$ is the symmetric part of $\mathbf{M}$ and $j=$ 2, 3, 4, 5 and therefore from Eq. (28) it follows that

$$
\dot{W}=-\Phi^{T} M_{1} \Phi \leq 0 .
$$

The stability of $\tilde{v}=0, \tilde{z}=0$ and $\tilde{\Theta}=\mathbf{0}$ follows. Using Barbalat's Lemma it is possible to show that $\lim _{t \rightarrow \infty} \tilde{v}(t)=0$. Convergence of $\tilde{z}=0$ and $\tilde{\Theta}=\mathbf{0}$ can not be guaranteed if there is no persistence of excitation. In this case the equilibria that are reached satisfy

$$
\begin{aligned}
\tilde{\sigma}_{0} \hat{z}\left(1-w_{1} / \sigma_{0}\right)-\hat{z} f\left(v_{r}\right) \tilde{\sigma}_{3}+\hat{v}_{r} \tilde{\sigma}_{4} & =0 \\
\tilde{z}+\frac{\hat{z}}{\sigma_{0}} \tilde{\sigma}_{0} & =0
\end{aligned}
$$

Remark 3. In the above combined stability analysis the controller Lyapunov candidate $W_{4}$ was not included because the controlled target error $\tilde{s}$ given by (17) is decoupled with observer and parameter adaptation errors $\tilde{\Theta}, \tilde{z}$, and $\tilde{v}$.

\section{UNDERESTIMATION OF FRICTION COEFFICIENT}

A very desirable feature to be attained with the observer and adaptive scheme in Eqs. (5), (15) and (11) is the underestimation of the maximum coefficient of friction, $\mu_{m}$. This underestimation provides conservative estimates for intervehicle distance that will yield safe emergency braking maneuvers.

From Eq. (8) it is clear that

$$
\tilde{\sigma}_{0}>0, \quad \tilde{\sigma}_{3}<0, \quad \text { and } \quad \tilde{\sigma}_{4}>0 .
$$

will produce this desired underestimation of $\mu_{m}$, i.e. $\hat{\mu}_{m} \leq \mu_{m}$ provided that

(1) the estimated state variables $\hat{v}$ and $\hat{z}$ converge to the true states fast and

(2) $z \geq 0, v_{r} \geq 0$ and $f\left(v_{r}\right) \geq 0$.

In this section it is assumed that $\tilde{\sigma}_{0}(0)>0$, $\tilde{\sigma}_{3}(0)<0$ and $\tilde{\sigma}_{4}(0)>0$ are selected and that $\tilde{v}=$ 0 . Furthermore it is assumed that $\tilde{z}(0)$ satisfies Eq. (30). Under these assumptions, the structure of the system composed by $\tilde{\sigma}_{0}, \tilde{\sigma}_{3}$ and $\tilde{\sigma}_{4}$ is

$$
\left[\begin{array}{c}
\dot{\tilde{\sigma}}_{0} \\
\dot{\tilde{\sigma}}_{3} \\
\dot{\tilde{\sigma}}_{4}
\end{array}\right]=\left[\begin{array}{ccc}
-\alpha \gamma_{0} \hat{z}^{2} & \gamma_{0} f\left(v_{r}\right) \hat{z}^{2} & -\gamma_{0} \hat{z} v_{r} \\
\alpha \gamma_{3} f\left(v_{r}\right) \hat{z}^{2} & -\gamma_{3} f^{2}\left(v_{r}\right) \hat{z}^{2} & \gamma_{3} f\left(v_{r}\right) \hat{z} v_{r} \\
-\alpha \gamma_{4} \hat{z} v_{r} & \gamma_{4} f\left(v_{r}\right) \hat{z} v_{r} & -\gamma_{4} v_{r}^{2}
\end{array}\right]\left[\begin{array}{c}
\tilde{\sigma}_{0} \\
\tilde{\sigma}_{3} \\
\tilde{\sigma}_{4}
\end{array}\right]
$$

with $\alpha=1-\frac{\sigma_{0}-\sigma_{3} f\left(v_{r}\right)}{\sigma_{0}}$. For analysis purposes consider the system in Eq. (32) as time invariant, the solution, with initial conditions $\tilde{\sigma}_{0}(0), \tilde{\sigma}_{3}(0)$ and $\tilde{\sigma}_{4}(0)$, is

$$
\begin{aligned}
\tilde{\sigma}_{0}(t)= & \frac{1}{\beta}\left[\left(\alpha \gamma_{0} \hat{z}^{2} e^{-\beta t}+\gamma_{3} \hat{z}^{2} f^{2}\left(v_{r}\right)+\gamma_{4} v_{r}^{2}\right) \tilde{\sigma}_{0}(0)\right. \\
& +\left(1-e^{-\beta t}\right) \gamma_{0} \hat{z}^{2} f\left(v_{r}\right) \tilde{\sigma}_{3}(0) \\
& \left.+\left(1-e^{-\beta t}\right) \gamma_{4} v_{r}^{2} \tilde{\sigma}_{4}(0)\right] \\
\tilde{\sigma}_{3}(t) & =\frac{1}{\beta}\left[\left(1-e^{-\beta t}\right) \alpha \gamma_{3} \hat{z}^{2} f\left(v_{r}\right) \tilde{\sigma}_{0}(0)\right. \\
& +\left(\alpha \gamma_{0} \hat{z}^{2}+\gamma_{4} v_{r}^{2}+\gamma_{3} \hat{z}^{2} f^{2}\left(v_{r}\right) e^{-\beta t}\right) \tilde{\sigma}_{3}(0) \\
& \left.-\left(\left(1-e^{-\beta t}\right) \gamma_{3} \gamma_{4} v_{r}^{2} / \gamma_{0}\right) \tilde{\sigma}_{4}(0)\right] \\
\tilde{\sigma}_{4}(t) & =\frac{1}{\beta}\left[\left(1-e^{-\beta t}\right) \alpha \gamma_{0} \hat{z}^{2} \tilde{\sigma}_{0}(0)\right. \\
& -\left(1-e^{-\beta t}\right) \gamma_{0} \hat{z}^{2} f\left(v_{r}\right) \tilde{\sigma}_{3}(0) \\
+\left(\alpha \gamma_{0}\right. & \left.\left.\hat{z}^{2}+\gamma_{3} \hat{z}^{2} f^{2}\left(v_{r}\right)+\gamma_{4} v_{r}^{2} e^{-\beta t}\right) \tilde{\sigma}_{4}(0)\right] \quad(33 \mathrm{c}) \\
\text { where } \beta & =\alpha \gamma_{0} \hat{z}^{2}+\gamma_{3} \hat{z}^{2} f^{2}\left(v_{r}\right)+\gamma_{4} v_{r}^{2} .
\end{aligned}
$$

Consider now the following assumption and lemma

Assumption 4.

$$
\begin{array}{ll}
\text { a) } & v(t) \geq v_{\min } ; \forall t \geq 0 \\
\text { b) } \sigma_{0}-\sigma_{3} f\left(v_{r}\right) \geq 0 ; \forall v_{r}>0
\end{array}
$$

Lemma 5. Assume that $\tilde{\sigma}_{0}(0)>0, \tilde{\sigma}_{3}(0)<0$ and $\tilde{\sigma}_{4}(0)>0$ are choosen and that lemma 1 and assumption 4 hold, then there exist gains $\gamma_{0}, \gamma_{3}$ and $\gamma_{4}$ such that if the following conditions are satisfied

$$
\begin{aligned}
& \gamma_{4} v_{r}^{2} \tilde{\sigma}_{0}(0)>\gamma_{0} \hat{z}^{2} f\left(v_{r}\right)\left|\tilde{\sigma}_{3}(0)\right| \\
&\left(\alpha \gamma_{0} \hat{z}^{2}+\gamma_{4} v_{r}^{2}\right)\left|\tilde{\sigma}_{3}(0)\right|>\alpha \gamma_{3} \hat{z}^{2} f\left(v_{r}\right) \tilde{\sigma}_{0}(0)
\end{aligned}
$$

then $\tilde{\sigma}_{0}(t) \geq 0, \tilde{\sigma}_{3}(t) \leq 0$ and $\tilde{\sigma}_{4}(t) \geq 0 ; \forall t \geq 0$.

Proof: First assume that $t$ is close to 0 , then the evolution of $\tilde{\sigma}_{0}(t), \tilde{\sigma}_{3}(t)$ and $\tilde{\sigma}_{4}(t)$ is dominated by $\tilde{\sigma}_{0}(0)>0, \tilde{\sigma}_{3}(0)<0$ and $\tilde{\sigma}_{4}(0)>0$ because the term $\left(1-e^{-\beta t}\right)$ can be neglected. Now assume the worst possible case, that will happen if $t \gg 0$. In this situation for $\tilde{\sigma}_{0}(t)$ to remain positive, according to Eq. (33a), it is necessary that

$$
\begin{aligned}
& \left(\gamma_{3} \hat{z}^{2} f^{2}\left(v_{r}\right)+\gamma_{4} v_{r}^{2}\right) \tilde{\sigma}_{0}(0)+\gamma_{4} v_{r}^{2} \tilde{\sigma}_{4}(0) \\
& >\gamma_{0} \hat{z}^{2} f\left(v_{r}\right)\left|\tilde{\sigma}_{3}(0)\right|
\end{aligned}
$$

Ineq. (38) will hold if

$$
\gamma_{4} v_{r}^{2} \tilde{\sigma}_{0}(0)>\gamma_{0} \hat{z}^{2} f\left(v_{r}\right)\left|\tilde{\sigma}_{3}(0)\right|
$$

that is precisely Ineq. (36). Similarly, according to Eq. $(33 \mathrm{~b})$, for $\tilde{\sigma}_{3}(t)$ to remain negative it is necessary that

$$
\begin{aligned}
& \left(\alpha \gamma_{0} \hat{z}^{2}+\gamma_{4} v_{r}^{2}\right)\left|\tilde{\sigma}_{3}(0)\right|+\frac{\gamma_{3} \gamma_{4}}{\gamma_{0}} v_{r}^{2} \tilde{\sigma}_{4}(0) \\
& >\alpha \gamma_{3} \hat{z}^{2} f\left(v_{r}\right) \tilde{\sigma}_{0}(0)
\end{aligned}
$$

Ineq. (40) will hold in turn if

$$
\left(\alpha \gamma_{0} \hat{z}^{2}+\gamma_{4} v_{r}^{2}\right)\left|\tilde{\sigma}_{3}(0)\right|>\alpha \gamma_{3} \hat{z}^{2} f\left(v_{r}\right) \tilde{\sigma}_{0}(0)
$$

that is Ineq. (37). According to Eq. $(33 \mathrm{c}), \tilde{\sigma}_{4}(0)$ will remain always positive.

Finally, the main result of this paper is stated in the following theorem 
Theorem 6. Consider assumption 4 and Lemmas 1-5, then under the observer and adaption laws in Eq. (4), (10) and (11) the equilibrium $\tilde{v}=$ $0, \tilde{z}=0$ and $\tilde{\Theta}=\mathbf{0}$ is stable. Moreover the maximum coefficient of friction $\mu_{m}$ is underestimated and $\lim _{t \rightarrow \infty} \tilde{v}(t)=0, \lim _{t \rightarrow \infty} \tilde{z}(t)=0$ and $\lim _{t \rightarrow \infty} \tilde{\Theta}=\mathbf{0}$.

Proof: The choice of $\tilde{z}(0)<0, \tilde{\sigma}_{0}(0)>0$, $\tilde{\sigma}_{3}(0)<0$ and $\tilde{\sigma}_{4}(0)>0$ together with Lemma 5 implies that $\hat{\mathbf{U}} \tilde{\Theta} \geq 0$ and therefore that that the product $\tilde{\Theta}^{T} \hat{\mathbf{U}}^{T} \hat{\mathbf{U}} \tilde{\tilde{\Theta}}$ does not vanish, except when $\tilde{\Theta}=\mathbf{0}$.

Choose Lyapunov function candidate $V$ as

$$
V=h_{1} W_{1}+h_{2} W_{2}+h_{3} W_{3}=\sum_{i=1}^{3} h_{i} W_{i}
$$

with $h_{1}, h_{2}$ and $h_{3}$ positive scalars. The time derivative of Eq. (42) can be bounded by

$$
\dot{V} \leq-\frac{1}{2} \Psi^{T}\left(\mathbf{H S}+\mathbf{S}^{T} \mathbf{H}\right) \Psi
$$

where $\Psi=[\|\tilde{\Theta}\||\tilde{z}||\tilde{v}|]^{T}, \mathbf{H}=\operatorname{diag}\left\{h_{3}, h_{2}, h_{1}\right\}$ and

$$
\mathbf{S}=\left[\begin{array}{ccc}
\left\|\hat{\mathbf{U}}^{T} \hat{\mathbf{U}}\right\| & \left\|\hat{\mathbf{U}}^{T} \mathbf{U}_{2} \Theta\right\| & \left\|\hat{\mathbf{U}}^{T} \mathbf{U}_{1} \Theta\right\| \\
\left\|\mathbf{U}_{3}\right\| & \sigma_{0} f\left(v_{r}\right) & -\left(1-\sigma_{0} f^{\prime}\left(v_{r}\right) \hat{z}\right) \\
\mathbf{0} & 0 & d(1-L)(v+\hat{v})
\end{array}\right]
$$

According to (Khalil, 1996) a necessary and sufficient condition for the existence of scalar $h_{1}, h_{2}$ and $h_{3}$ that will make Eq. (43) negative definite is that the principal minors of the matrix $\mathbf{S}$ are positive definite. The first two minors are proven directly to be positive, the third one is given by

$$
d(1-L)(v+\hat{v}) \hat{z}^{2} f^{2}\left(v_{r}\right) \sigma_{3} \geq 0
$$

provided that conditions on Lemmas 1 and 5 are satisfied. This proves aymptotic stability and therefore that $\lim _{t \rightarrow \infty} \tilde{v}(t)=0, \lim _{t \rightarrow \infty} \tilde{z}(t)=0$ and $\lim _{t \rightarrow \infty} \tilde{\Theta}=\mathbf{0}$.

The underestimation of $\mu_{m}$ follows directly from Eq. (8) and Lemma 5.

\section{SIMULATION RESULTS}

In this section the design of the previous section is tested. In the simulation, the parameters are taken from the LeSabre cars used in the California PATH program: $M=1701.0 \mathrm{Kg}, \mathrm{Ca}=0.3693 \mathrm{~N}$. $s^{2} / m^{2}, J=2.603 \mathrm{Kg} \cdot \mathrm{m}^{2}, R=0.323 \mathrm{~m}$ and $K_{b}=0.9$ for nominal values. Wheel angular velocity and vehicle longitudinal acceleration are used to design an observer-based emergency braking controller. Fig. 1 shows the vehicle velocity, friction coefficient and braking pressure and dynamic surface value during the emergency braking maneuver. Fig. 2 illustrates the estimated internal state error $\tilde{z}$ and vehicle velocity error $\tilde{v}$. Fig. 3 shows the estimated friction parameters $\sigma_{0}, \sigma_{3}$ and $\sigma_{4}$ and the coefficent of friction estimation error $\tilde{\mu}$. During the simulation these parameters converged quickly to their true values. At the end of the maneuver there is a slight loss of convergence in the parameters value that obeys to the transition from dynamic to static friction. Convergence is not recovered rapidly because the updating term in the adaption law is quite small at this time. This situation can be avoided by turning the adaptation off.
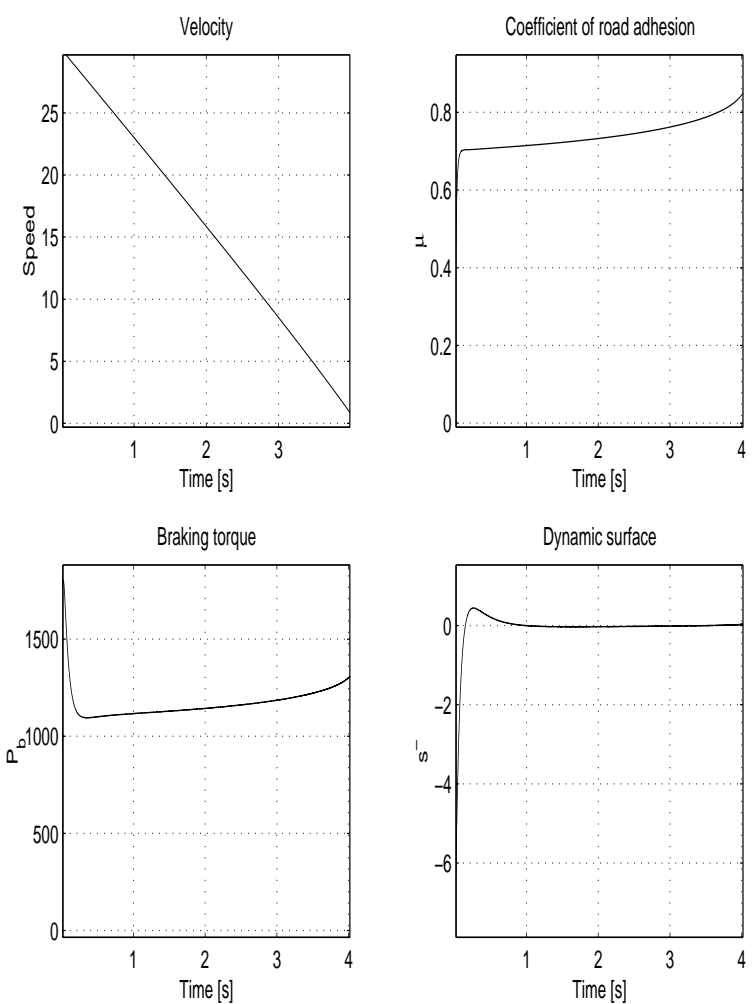

Fig. 1. Velocity $v$, friction coefficient $\mu$ and braking pressure $P_{b}(K P a)$ and dynamic surface.
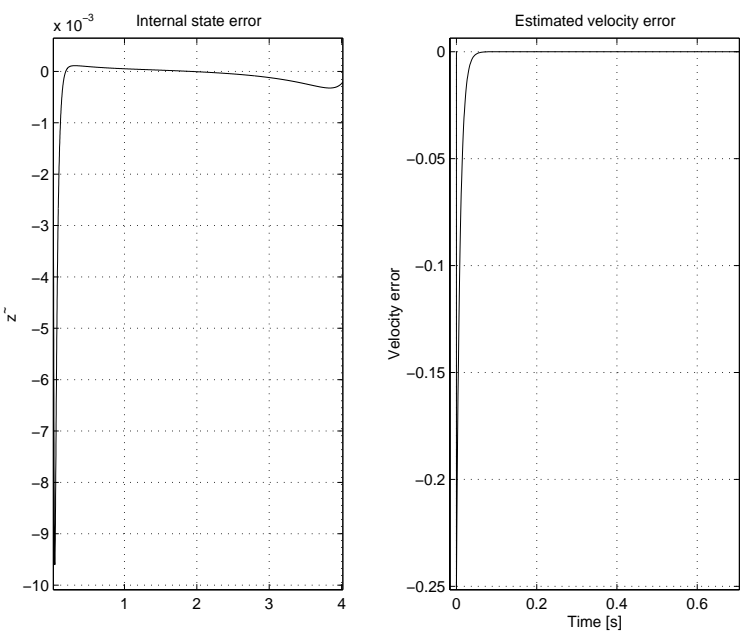

Fig. 2. Internal state and velocity estimation errors. 

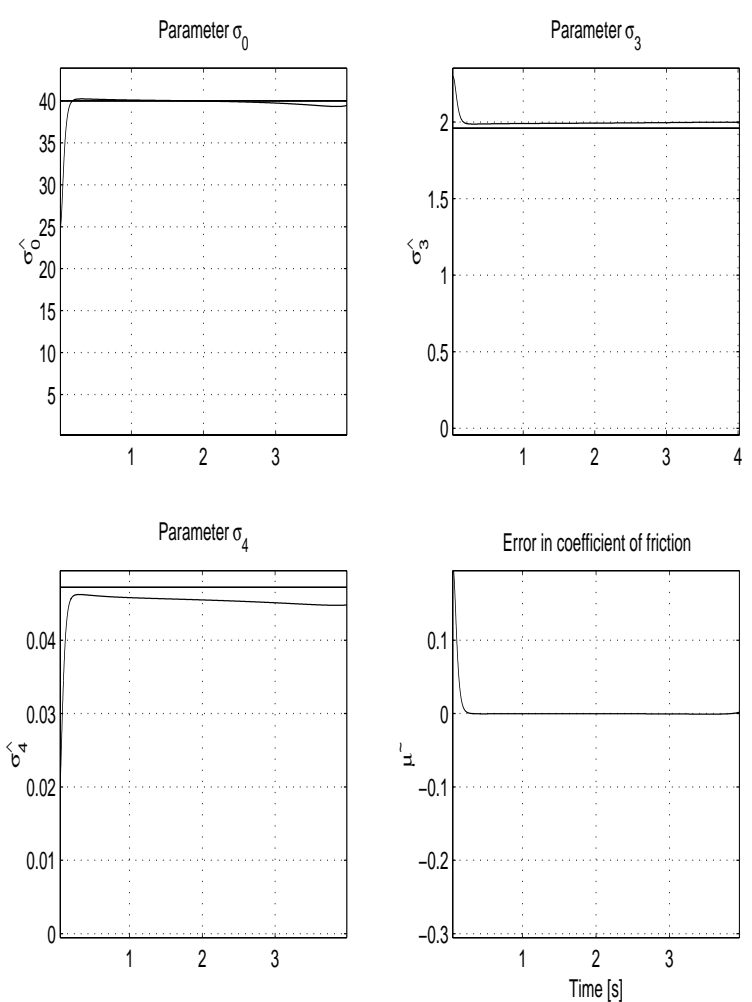

Fig. 3. Adapted parameters and error in coefficient of friction.

\section{CONCLUSIONS}

In this paper the emergency braking of vehicles under unknown tire/road conditions and system states, based on a dynamical friction model is discussed. It is assumed that measurements of wheel angular velocity and longitudinal acceleration are available. The braking pressure controller is determined based on the estimation of system state and the friction parameters. The asymptotic convergence of the estimated states and parameter estimates has been proven, under proper selection of adaptation gains and initial estimation errors. The simulation results show that, by applying this controller, the vehicle can be stopped quickly with near maximum deceleration.

\section{REFERENCES}

Alvarez, L., J. Yi, R. Horowitz and L. Olmos (2000). Emergency braking control with underestimation of friction coefficient. In: Proceedings of the 2000 American Control Conference. pp. 574-579.

Bakker, E., L. Nyborg and H.B. Pacejka (1987). Tyre modelling for use in vehicle dynamic studies. Society of Automotive Engineers Paper \# 870421.

Canudas, C., H. Olsson, K. J. Åstrom and P. Lischinsky (1995). A new model for control of systems with friction. IEEE Transactions on Automatic Control 40(3), 419-425.
Canudas de Wit, Carlos and Panagiotis Tsiotras (1999). Dynamic Tire Friction Models for Vehicle Traction Control. In: Proceedings of $38^{\text {th }}$ IEEE Conference of Decision and Control. Phoenix, AZ.

Canudas de Wit, Carlos and Roberto Horowitz (1999). Observers for Tire/Road Contact Friction using only wheel angular velocity information. In: Proceedings of $38^{\text {th }}$ IEEE Conference of Decision and Control. Phoenix, AZ.

Gerdes, J.C. and K.J. Hedrick (1995). Brake System Requirements for Platooning on an $\mathrm{Au}-$ tomated Highway. In: The American Control Conference. Seattle, Washington. pp. 165169.

Khalil, H.K. (1996). Nonlinear Systems. 2nd ed.. Prentice Hall. NJ.

Kiencke, U. (1993). Realtime estimation of adhesion characteristic between tyres and road. In: Proceedings of the IFAC World Congress. Vol. 1.

Wong, J. Y. (1993). Theory of Ground Vehicles. 2nd ed.. John Wiley \& Sons. New York, NY.

Yi, J., L. Alvarez, R. Horowitz and C. Canudas (2000). Adaptive emergency braking control based on a tire/road friction dynamic model. In: Proceedings of the 2000 Conference on Decision and Control. pp. 456-461.

Yi, J., L. Alvarez, X. Claeys, R. Horowitz and C. Canudas (2001). Adaptive observer-based emergency braking/traction control using dynamic tire/road friction model. In: Proceedings of the American Control Conference. pp. 19-24. 